\title{
Micromachined Waveguide Interposer for the Characterization of Multi-port Sub-THz Devices
}

\section{Adrian Gomez-Torrent ${ }^{1}$ (D) . Joachim Oberhammer ${ }^{1}$}

Received: 16 October 2019 / Accepted: 12 December 2019/Published online: 8 January 2020

(C) The Author(s) 2020

\begin{abstract}
This paper reports for the first time on a micromachined interposer platform for characterizing highly miniaturized multi-port sub-THz waveguide components. The reduced size of such devices does often not allow to connect them to conventional waveguide flanges. We demonstrate the micromachined interposer concept by characterizing a miniaturized, three-port, 220-330-GHz turnstile orthomode transducer. The interposer contains low-loss micromachined waveguides for routing the ports of the device under test to standard waveguide flanges and integrated micromachined matched loads for terminating the unused ports. In addition to the interposer, the measurement setup consists of a micromachined square-to-rectangular waveguide transition. These two devices enable the characterization of such a complex microwave component in four different configurations with a standard two-port measurement setup. In addition, the design of the interposer allows for independent characterization of its sub-components and, thus, for accurate de-embedding from the measured data, as demonstrated in this paper. The measurement setup can be custom-designed for each silicon micromachined device under test and co-fabricated in the same wafer due to the batch nature of this process. The solution presented here avoids the need of CNC-milled test-fixtures or waveguide pieces that deteriorate the performance of the device under test and reduce the measurement accuracy.
\end{abstract}

Keywords Waveguide $\cdot$ Silicon micromachining $\cdot$ Terahertz $\cdot$ Multi-port · Measurement · Interposer · Test-fixture · DRIE · Orthomode transducer

Adrian Gomez-Torrent

adriango@kth.se

1 Department of Micro and Nanosystems, School of Electrical Engineering and Computer

Science, KTH Royal Institute of Technology, Stockholm SE-100 44, Sweden 


\section{Introduction}

Waveguide components working in the millimeter-wave $(\mathrm{mmW})$ and sub-mmW frequency ranges of the electromagnetic spectrum are of particular interest for many applications due to their high performance and reduced size [1]. Silicon micromachining of waveguide components and systems in this frequency range enables high-complexity, high-performance, and drastically miniaturized devices [24]. Although silicon-micromachined waveguide devices are usually substantially smaller than waveguide flanges, it is often necessary to connect them to such flanges in specific system architectures, or solely for their characterization.

A smart design can allow for the direct mounting of highly miniaturized micromachined components between two waveguide flanges, if axial and opposite-face port arrangement is featured, as demonstrated for micromachined filters $[5,6]$ or switches $[7,8]$. However, such direct connection cannot be used for devices with an off-axis port arrangement or multi-port devices if the device is smaller than the area required for the individual flanges.

The conventional solution to characterize sub- $\mathrm{THz}$ devices, or to utilize them in systems with internal flange connections, is to manufacture them in a CNC-milled split-block configuration [9,10], or to mount micromachined devices in CNC-milled test fixtures [3, 11-13]. Such CNC-milled parts can be costly and difficult to fabricate, and significantly add to the overall losses of the system due to inferior manufacturing tolerances and the long waveguide sections required [3].

Another common approach for characterizing multi-port devices with a two-port measurement system is to co-fabricate multiple devices in different port configurations. For each configuration, two of the ports are accessible to standard waveguide flanges and the remaining ports are, for instance, terminated with integrated absorbers $[12,14,15]$. This avoids the need for long waveguide sections to re-route each port but requires the fabrication of several prototypes that should be almost identical, as it is otherwise not possible to extract accurate S-parameters. Moreover, the loads in these measurement setups are integrated into the component and cannot be characterized individually, thus their non-ideal behavior cannot be properly deembedded. This, together with the fact that the fabricated devices are not identical, results in significant uncertainty in the measured S-parameters.

Silicon micromachining is an enabling fabrication technology for sub-THz waveguide devices [1-4, 8, 11, 12, 16-18]. These cited works have demonstrated complex and low-loss waveguide geometries, MEMS integration, or system-on-chip architectures. The authors have recently introduced a very low-loss micromachined waveguide technology $(0.02-0.07 \mathrm{~dB} / \mathrm{mm}$ in the $220-330-\mathrm{GHz}$ band, [17]), which is a near-ideal candidate for routing waveguide signals in micromachined test fixtures.

In this paper, we report for the first time on a measurement setup for the characterization of miniaturized multi-port waveguide components at sub- $\mathrm{THz}$ frequencies. The silicon-micromachined interposer setup was used for the first time to characterize a recently published turnstile orthomode transducer (OMT) in the $220-330-\mathrm{GHz}$ frequency range [4]. The setup is composed of a silicon-micromachined interposer and a square to rectangular waveguide transition, which replaces the alternative nonoptimal CNC-milled test fixtures. Besides a low-loss micromachined waveguide 
routing network, the interposer contains integrated micromachined loads to terminate the unused ports in specific measurement configurations. This paper describes the concept of the measurement setup, the interposer design with the integrated micromachined matched-loads, reports on the characterization of all individual subcomponents, and discusses the effect of their performance on the characterization of the device under test (DUT).

\section{Measurement Requirements and Interposer Concept}

The port arrangement of the turnstile OMT (the DUT) in [4] is shown in Fig. 1. The DUT has a centered square waveguide port on one side and two rectangular waveguide ports (vertical and horizontal polarization) in a non-axial, but rotationally symmetric, arrangement on the opposing face. Due to the reduced size of the OMT $(5 \mathrm{~mm} \times 5 \mathrm{~mm} \times 0.9 \mathrm{~mm})$, all three waveguide ports are located within a distance of $4 \mathrm{~mm}$, i.e., an area 16 times smaller than the standard flange footprint. Moreover, the square port in the DUT carries the two orthogonal polarizations, so it consists of two independent electrical ports (P1 and P2 in Fig. 1) that have to be accessed with the vector network analyzer (VNA) during the measurements.

The characterization of this device requires routing the rectangular ports (P3 and P4 in Fig. 1) with an offset of $20 \mathrm{~mm}$ (size of a standard test-port flange), and to independently access ports $\mathrm{P} 1$ and $\mathrm{P} 2$ in the square waveguide. Furthermore, to characterize the device in a two-port measurement setup, the test fixture must offer the possibility of terminating the unused rectangular port in the different measurement configurations.

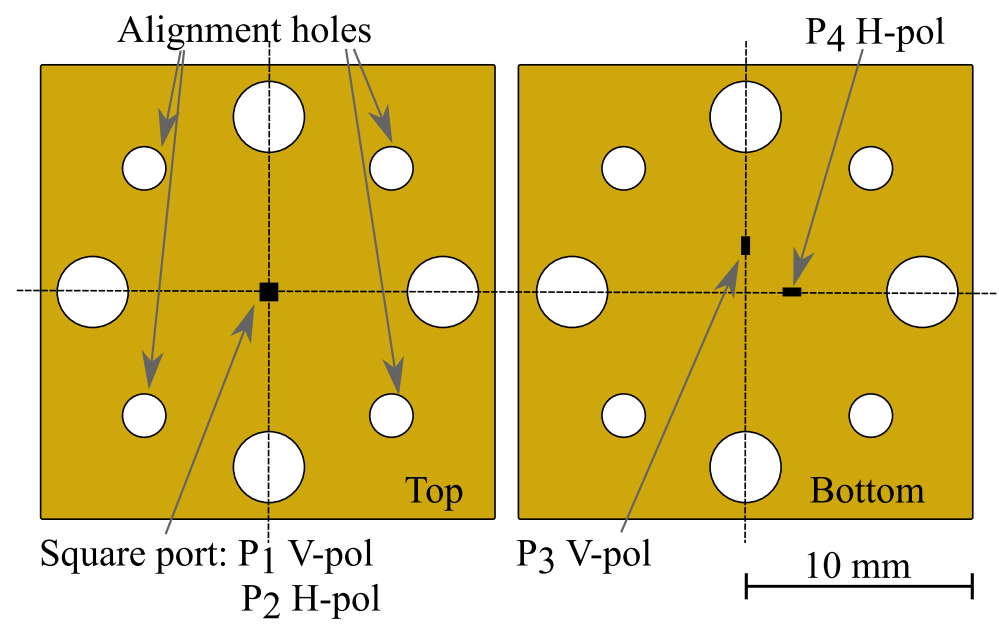

Fig. 1 Device under test: The OMT-chip top and bottom face layouts with a non-axial port arrangement, including micromachined alignment holes and holes for the waveguide screws according to the UG-387 standard flange. The square port is located at the center of the chip, while the rectangular ports are in a rotationally symmetric arrangement at an offset to the square port on the opposing side 
The possibilities for characterizing different port configurations of the DUT are shown in Fig. 2, utilizing a silicon-micromachined multi-step square-to-rectangular waveguide transition and an interposer that routes one of the rectangular DUT ports to the VNA test port. The interposer also contains two integrated micromachined matched loads for terminating the third DUT port in different configurations, which were recently published by the authors [19]. Since the two rectangular ports are rotationally symmetric on the DUT, it is possible to connect either the vertical or the horizontal polarization port ( $\mathrm{P} 3$ or $\mathrm{P} 4$, respectively) of the DUT while the other port is loaded by one of the integrated matched loads in the interposer, simply by rotating the DUT through $90^{\circ}$, as shown in Fig. 2.

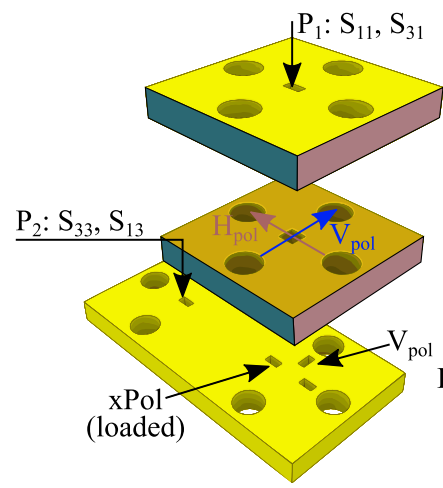

(a)
Rect./Square transition

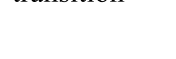

Interposer

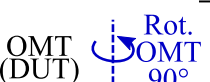
(DUT) $\begin{gathered}\text { OMT } \\ \text { SOMT }\end{gathered}$<smiles>[TeH2][TeH2]</smiles>

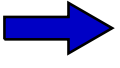

(1)

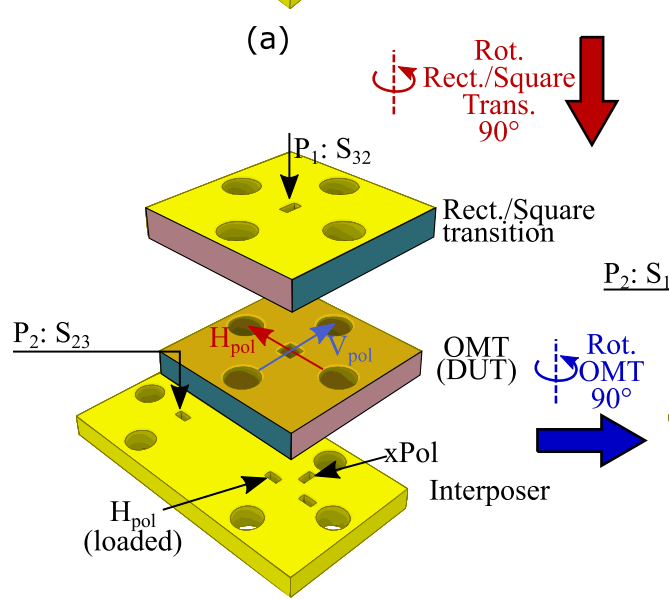

(c)

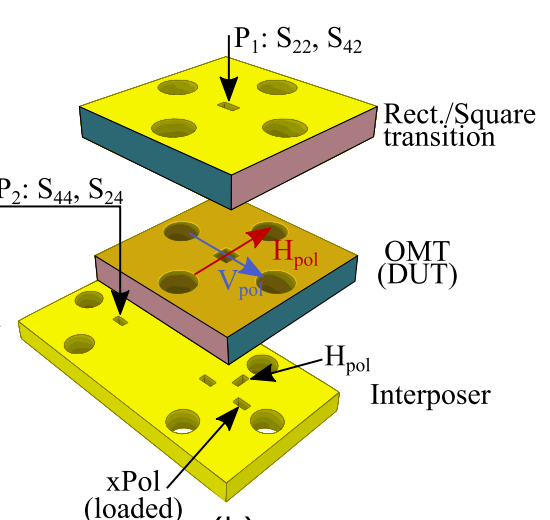

(b)

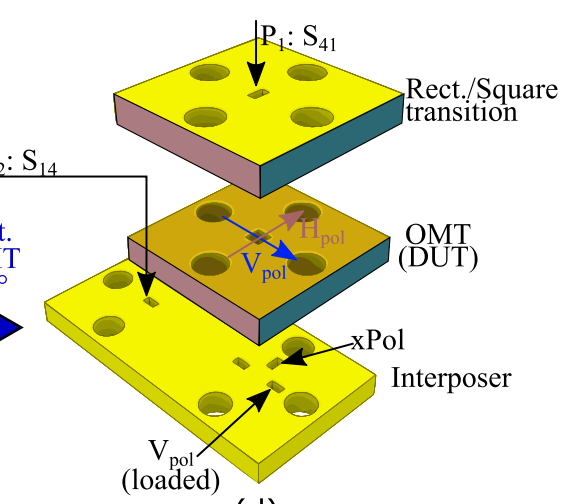

(d)

Fig. 2 Concept drawings of the four (a-d) configurations used during the measurements, showing the Sparameters extracted after de-embedding for each case. For changing from (a) to (b) configuration or for changing from (c) to (d) configuration, the rectangular-to-square waveguide transition is unchanged, but the OMT is rotated $90^{\circ}$. For changing from (a) to (c) or (b) to (d) configuration, the rectangular-to-square transition must be rotated $90^{\circ}$ 
The four measurement configurations are as follows:

(a) connect the V-pol channel in the square port and measure the V-pol rectangular port, $\mathrm{H}$-pol terminated by a matched load $\left(\mathrm{S}_{11}, \mathrm{~S}_{31}, \mathrm{~S}_{13}\right.$, and $\left.\mathrm{S}_{33}\right)$;

(b) connect the H-pol channel in the square port and measure the H-pol rectangular port, V-pol terminated by a matched load $\left(\mathrm{S}_{22}, \mathrm{~S}_{42}, \mathrm{~S}_{24}\right.$, and $\left.\mathrm{S}_{44}\right)$;

(c) connect the $\mathrm{H}$-pol channel in the square port and measure the V-pol rectangular port, $\mathrm{H}$-pol terminated by a matched load $\left(\mathrm{S}_{23}\right.$ and $\left.\mathrm{S}_{32}\right)$;

(d) connect the V-pol channel in the square port and measure the H-pol rectangular port, V-pol terminated by a matched load $\left(\mathrm{S}_{14}\right.$ and $\left.\mathrm{S}_{41}\right)$.

The waveguide section that routes the signal in the interposer can be independently characterized and thus de-embedded from the measurements. The de-embedding process allows for the extraction of all the relevant RF parameters of the single multiport DUT from the measured raw data of the different dual-port measurements. The lower uncertainty of microchip-to-metal flange interfaces as compared with metalto-metal-flange interfaces allow for significantly more accurate de-embedding of the DUT performance from the measurements. The tighter tolerances of the siliconto-metal connection relies on a novel alignment hole approach, demonstrated by Campion et al. by developing high-precision calibration shims for $\mathrm{THz}$ frequencies $[20,21]$.

\subsection{Interposer}

The silicon micromachined interposer shown in Fig. 3, with a $20 \mathrm{~mm} \times 40 \mathrm{~mm}$ footprint, is used to route the signals from ports P3 and P4 from the DUT to the VNA, according to the configurations shown in Fig. 2. Half of the chip area is utilized to connect to the flange of one of the VNA test ports. The other half accommodates the other test port flange, interfacing the DUT in two different positions allowing for the measurement of the return loss and insertion loss for both polarization channels and the cross-polarization of the OMT.

The interposer consists of two micromachined silicon-on-insulator (SOI) chips that are bonded together, as shown in the cross section in Fig. 4. The 20-mm-long waveguide section that routes the measurement signals and the pockets where the integrated loads are inserted (as described in [19]) are micromachined in chip 1 (see Fig. 3). Chip 2 forms the upper broad-wall of the H-plane split waveguides (as described in [17]) and contains the access ports. The total thickness of the interposer is $0.6 \mathrm{~mm}$, and each chip has three etched silicon layers that are used in the design for impedance matching. Both chips are co-fabricated in the same SOI wafer together with the DUT, and further fabrication details can be found in [4].

A photograph of the fabricated prototype is shown in Fig. 5. The dashed circles in Fig. 5a represent the positions where the standard waveguide flange can be mounted. The two offset mounting positions in the right part of the interposer are included to allow independent characterization of the waveguide before the DUT measurements. This feature is critical to accurately extract the S-parameters of the DUT from the two-port measurements. 


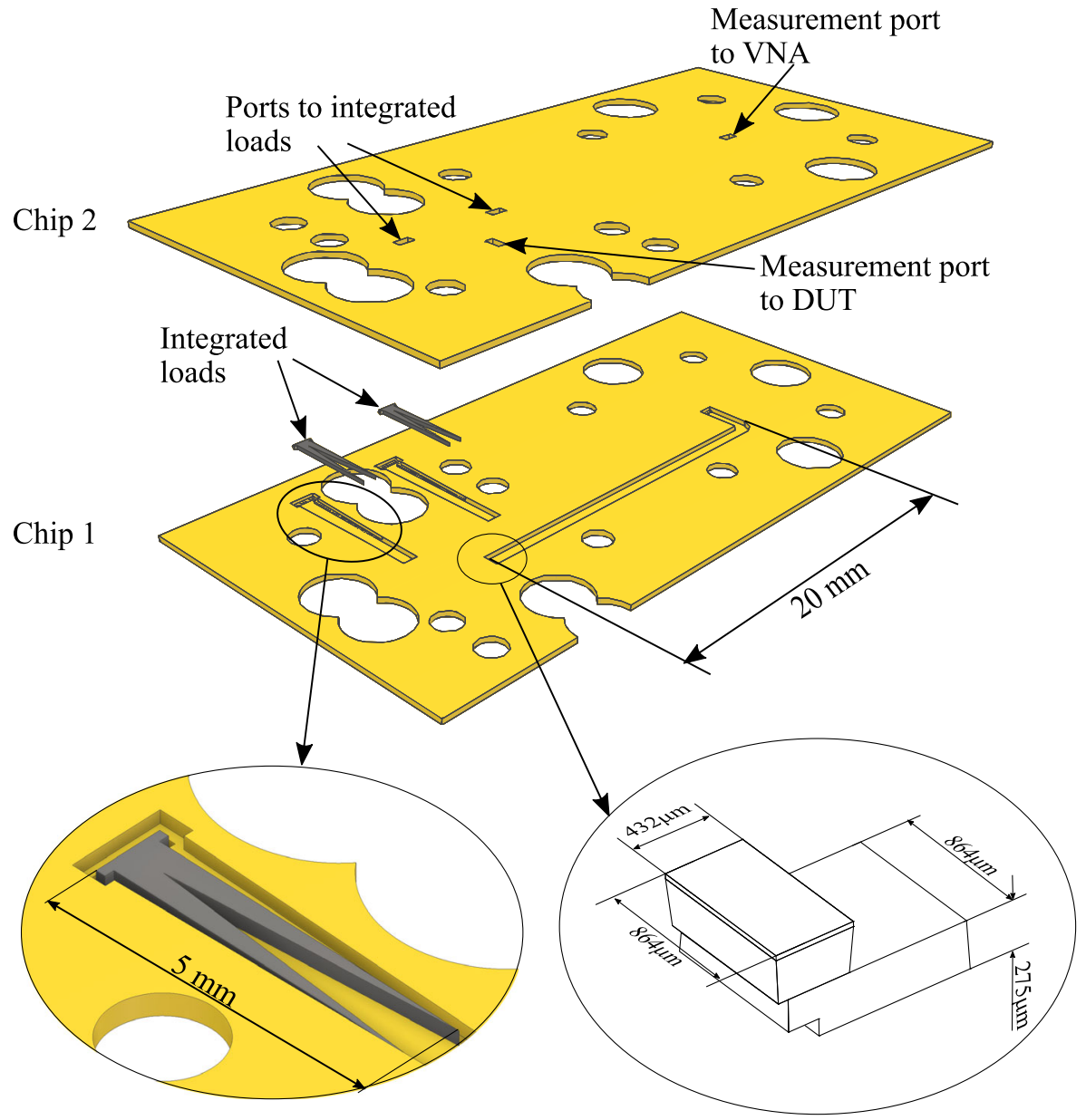

Fig. 3 Interposer CAD drawing showing the top (chip 2) and bottom (chip 1) chips. Chip 1 contains the waveguide section connecting the two measurement ports and the matched loads. Chip 2 forms the upper broad-wall of the waveguides and contains the different access ports. A close-up drawing of an integrated silicon-micromachined load is shown in the lower left inset, and a schematic drawing of the E-plane bend interfacing the in-plane reduced height waveguides with the out-of-plane standard size waveguides is shown in the lower right inset

The interposer mounted on one of the VNA ports is shown in Fig. 5b, which interfaces to two of the DUT ports. The flange to chip alignment accuracy is better than $5 \mu \mathrm{m}$, which is achieved by using a tight-fitting hole for one pin and an elliptical hole for the other pin, as reported by Campion et al. in [20].

\subsection{Rectangular-to-Square Waveguide Transition}

The rectangular-to-square transition shown in Fig. 6 is used to connect ports P1 and P2 (polarizations of the common square port) independently while setting a virtual 


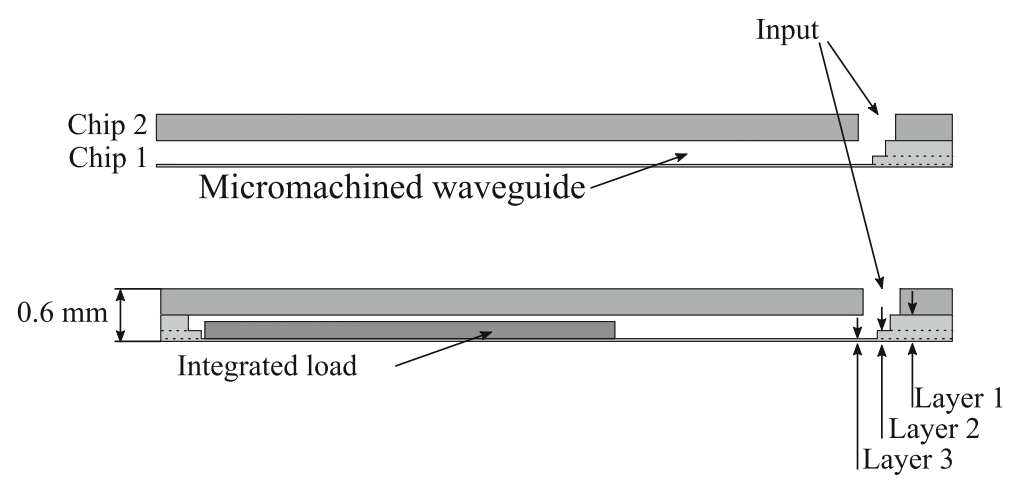

Fig. 4 Cross section showing the micromachined waveguides, the input E-plane bends, and the integrated loads inside the interposer

short-circuit to the other polarization port (waveguide in cut-off). Figure 2 shows how this transition can be mounted in two different positions to characterize either the return loss and insertion loss of a channel, or its cross-polarization.

The rectangular-to-square transition is built with three micromachined SOI chips, as shown in Fig. 6a, having a total size of $20 \mathrm{~mm} \times 20 \mathrm{~mm} \times 0.9 \mathrm{~mm}$. The high precision of the silicon micromachining process, together with the multi-step etching capability (described in [4]), enables the design of stepped transitions, as shown in Fig. 6 b. The cross section of the transition in Fig. 7 shows the arrangement of the three chips and the use of the different layers in the fabrication process to implement the complex 3D geometry shown in Fig. 6b. Stepped transitions can be extremely compact (shorter than $\lambda_{g}$ in the entire frequency range) while keeping high return loss (in this work, better than $25 \mathrm{~dB}$ in the entire band).

Once the transition is mounted on the test port, as shown in Fig. 6c, it provides a square-waveguide interface for the common port in the OMT and loads one of the two polarization channels, as shown in Fig. 6d, with mounted OMT on the transition. The loss and mismatching generated by the transition cannot be de-embedded

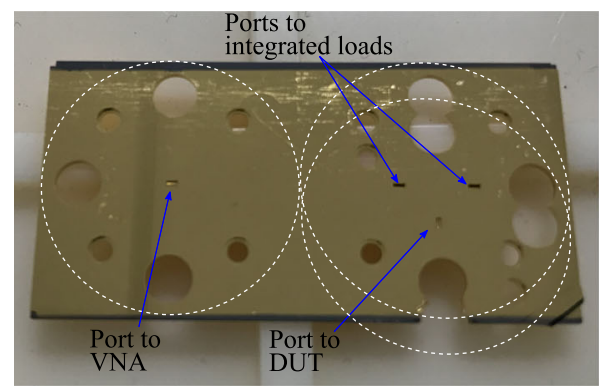

(a)

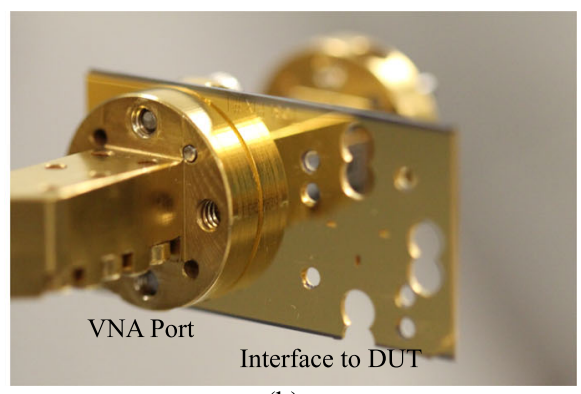

(b)

Fig. 5 Pictures of a fabricated silicon-micromachined interposer and $\mathbf{b}$ interposer mounted on the VNA test-port. White dashed lines represent the footprint of the waveguide flange in the different mounting positions 


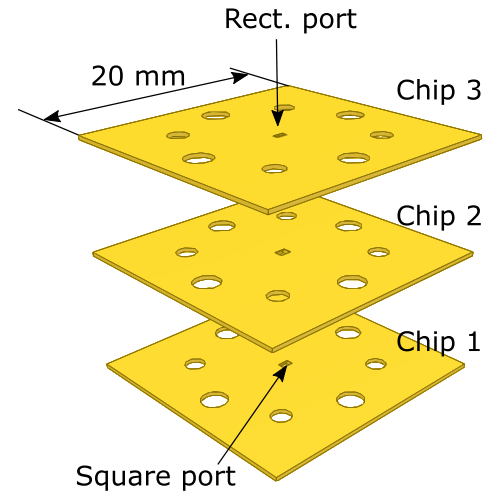

(a)

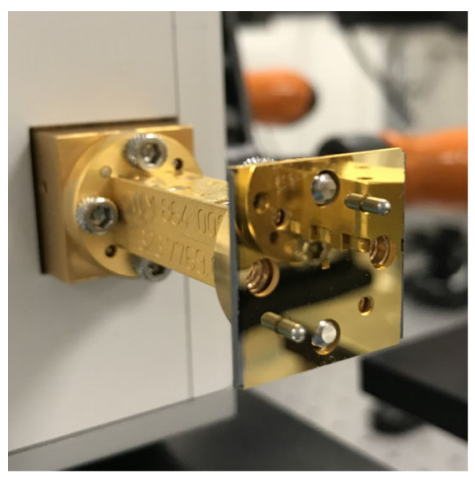

(c)

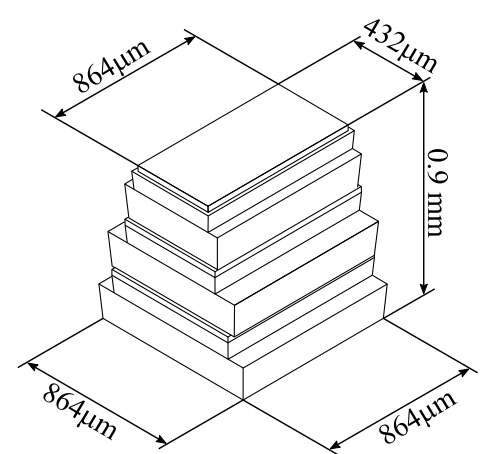

(b)

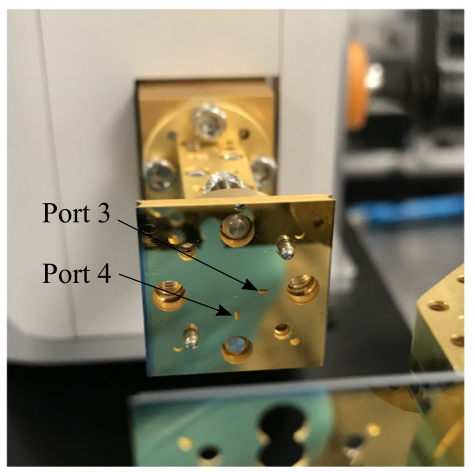

(d)

Fig. 6 Rectangular-to-square waveguide transition. a CAD drawing of the chip including alignment holes. b Detailed schematic drawing with waveguide dimensions. c Picture of the fabricated transition mounted on the test port. $\mathbf{d}$ Transition and OMT mounted on the test port

from the measured data since a square waveguide calibration kit would be necessary. Therefore, such compact micromachined transitions with low insertion loss and low standing wave ratio are beneficial to obtain reliable measurement results.

\section{Interposer Characterization}

The S-parameters of the individual sub-components in the interposer must be measured in order to de-embed their effects from the measurements and obtain the $\mathrm{S}$-parameters of the DUT. This is one of the main advantages of this technique as compared with the alternative approach of including the loads and long waveguide sections directly into the DUT, where their effect cannot be accurately deducted.

The interposer includes several holes that allow alignment of the test port to the different sub-components, as described in Section 2.1. The measured and simulated S-parameters of the 20-mm-long waveguide section inside the interposer are shown 


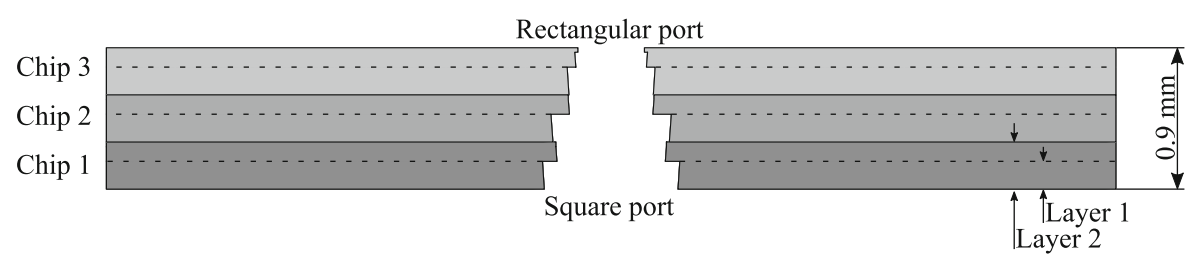

Fig. 7 Cross section of the square-to-rectangular waveguide transition. This compact design is achieved by using a multi-step DRIE process that enables a six-step transition by stacking three silicon-on-insulator chips

in Fig. 8. The measured insertion loss for the waveguide section ranges from 0.032 to $0.044 \mathrm{~dB} / \mathrm{mm}$, which is in good agreement with previously reported micromachined waveguides in the same frequency range [17].

The measured and simulated S-parameters of the integrated silicon micromachined loads (including the effect of the E-plane bends) are shown in Fig. 9. The input reflection level is in good agreement with the simulation data. Most of the reflections come from the input bends shown in Fig. 3 that were designed to use a minimum amount of chips; therefore, the return loss for these matched loads can be improved, as already shown in [19]. The purpose of the loads during the measurement of the OMT is to avoid strong resonances in the polarization channel not being measured. Therefore, the absorption provided by these loads is enough for this application, and mask space optimization was prioritized.

\section{DUT Measurements}

The measurement procedure of the DUT, a turnstile orthomode transducer, is described in Section 2. The four different configurations shown in Fig. 2 are needed for the characterization of most of the S-parameters of the device, i.e., the insertion loss, return loss, and cross-polarization for both channels. Isolation data $\left(\mathrm{S}_{12}, \mathrm{~S}_{21}\right.$,

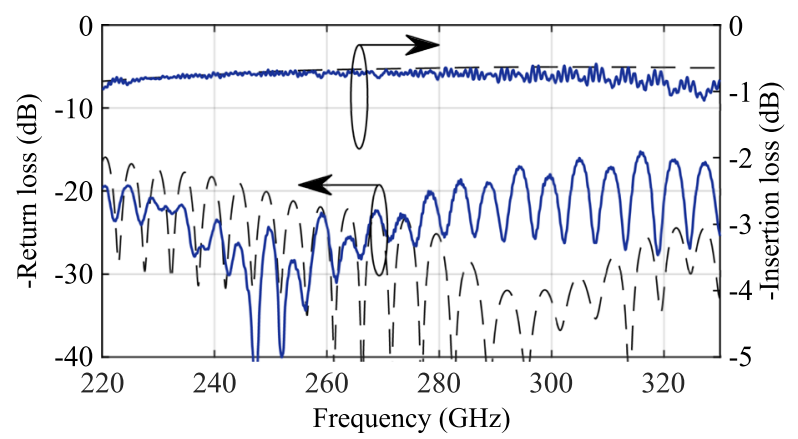

Fig. 8 Measured S-parameters of the waveguide section inside the interposer. Solid blue lines show measured data and black dashed lines show simulated data 


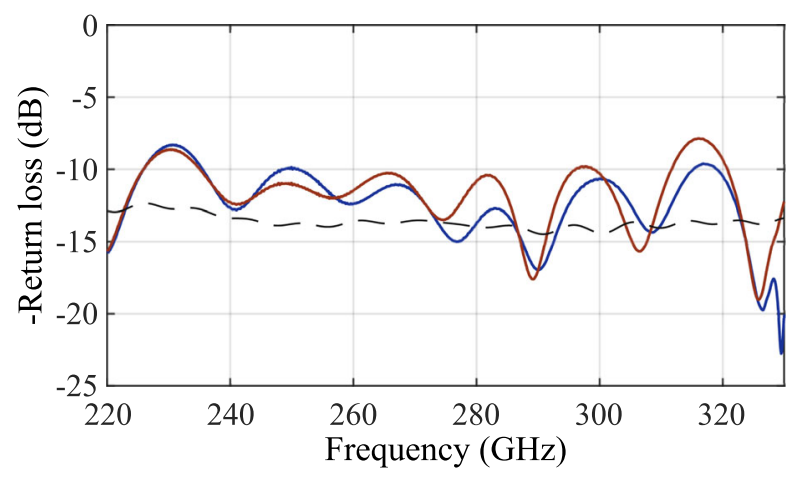

Fig. 9 Measured and simulated return loss of two integrated silicon loads, including the effect of the Eplane bends. Blue and red solid lines are the measured data for both loads and dashed grey lines show simulated data

$\mathrm{S}_{34}$, and $\mathrm{S}_{43}$ ) cannot be extracted using this measurement setup because ports $\mathrm{P} 1$ and P2 or P3 and P4 cannot be accessed simultaneously.

The setup during measurements is shown in Fig. 10. A Rohde \& Schwarz ZVA-24 VNA with ZC330 frequency extenders was used for the RF characterization of the component. The two VNA ports are calibrated using through, open, short, and match (TOSM) standards, which are measured to the reference plane that interfaces the silicon micromachined measurement setup. A $90^{\circ} \mathrm{H}$-plane bend is used to physically accommodate both frequency extenders, and two additional straight waveguide sections ("dummy pieces") are used to clamp the silicon chips using the screws in the waveguide flanges to a torque of $0.1 \mathrm{~N} \cdot \mathrm{m}$.

The de-embedding of the S-parameters of the waveguide section in the interposer is done using the inverse T-matrix method [22]. The extracted S-parameters from the DUT include the effect of the square-to-rectangular transition and assume a perfect load in the non-measured ports. This assumption is only valid if the non-measured

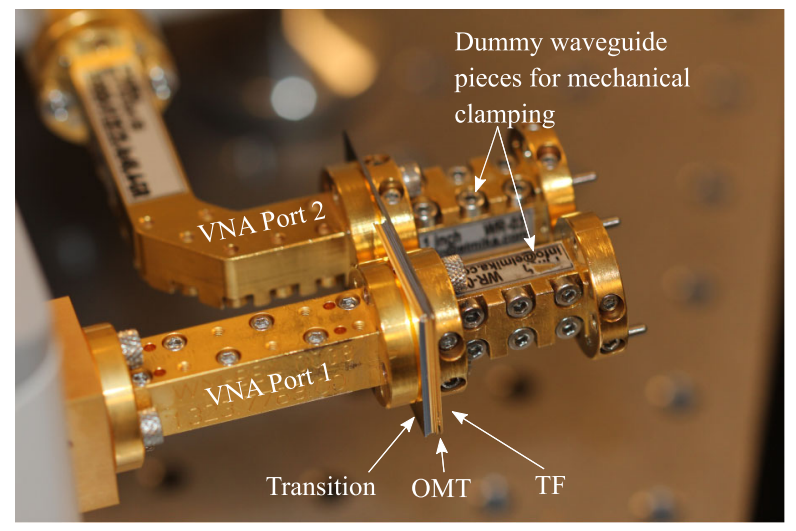

Fig. 10 Picture of the setup during measurements 


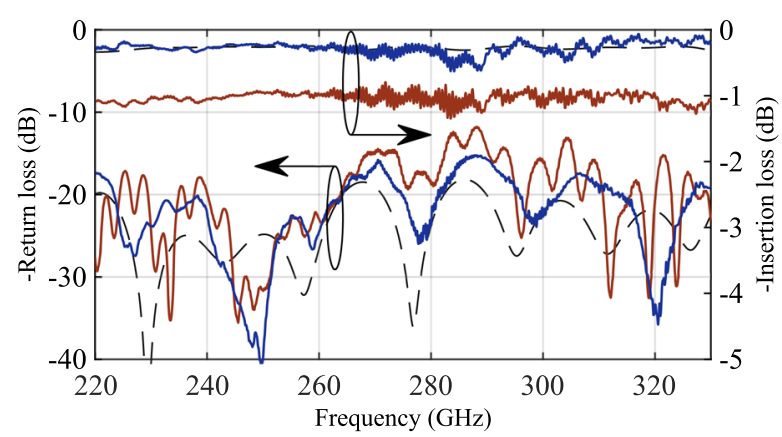

(a)

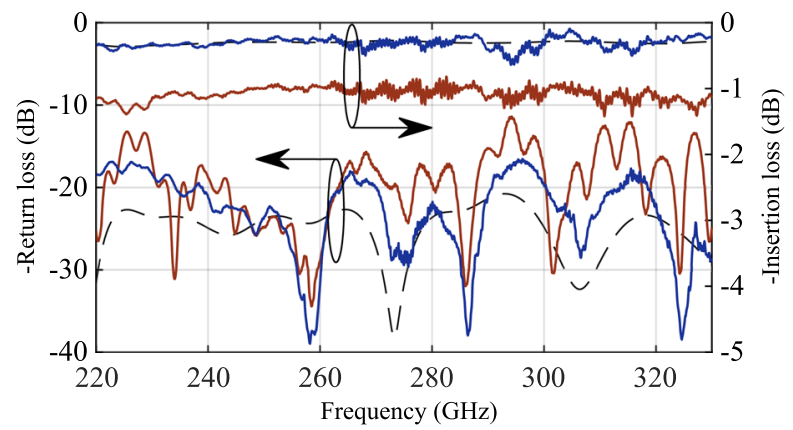

(b)

Fig. 11 Comparison between the measured raw data (solid red lines) and the extracted S-parameter of the OMT after de-embedding (solid blue lines) for the horizontal (a) and vertical (b) polarization channels. Black dashed lines show simulated S-parameters of the OMT

ports belong to the isolated channel, and the de-embedding process could not be done if low cross-polarization levels were observed. The measured cross-polarization for this OMT is always better than $35 \mathrm{~dB}$, as reported in [4]; therefore, this condition is met.

The comparison between the measured raw data, the de-embedded data, and the simulation data of the OMT for both polarization channels is plotted in Fig. 11. Both the ripple generated by the long waveguide section and the extra 1-dB insertion loss can be accurately subtracted from the measurement data by using the proposed setup. More detailed measurement results of the turnstile OMT can be found in [4].

\section{Conclusion}

A silicon micromachined interposer for the characterization of highly miniaturized multi-port waveguide components has been reported for the first time in this paper. This measurement setup provides lower loss, at a much lower cost, and better flangeto-chip alignment than conventional CNC-milled test fixtures, which provides better de-embedding accuracy. The higher accuracy, lower cost, ability to integrate loads, 
and versatility of silicon micromachined test interposers make them an enabling tool for the development of sub-THz waveguide components.

The entire test setup was manufactured simultaneously on the same wafer as the device under test, taking advantage of the batch processing nature of silicon micromachining, making possible the fabrication of custom-made measurement setups for every component. This solution was successfully tested for the characterization of a silicon micromachined wideband OMT, thus allowing for the first time, to the best of our knowledge, the characterization of a compact multi-port sub-THz waveguide component without the need of any CNC-milled flanges or fittings.

Funding Information Open access funding provided by Royal Institute of Technology. This work has received funding from the European Research Council (ERC) under the European Union's Horizon 2020 research and innovation programme (grant agreement no. 616846), and the Swedish Foundation for Strategic Research Synergy Grant Electronics SE13-007.

Open Access This article is distributed under the terms of the Creative Commons Attribution 4.0 International License (http://creativecommons.org/licenses/by/4.0/), which permits unrestricted use, distribution, and reproduction in any medium, provided you give appropriate credit to the original author(s) and the source, provide a link to the Creative Commons license, and indicate if changes were made.

\section{References}

1. G. Chattopadhyay, T. Reck, C. Lee, and C. Jung-Kubiak, Micromachined packaging for terahertz. systems, Proceedings of the IEEE 105 (2017), no. 6, 1139-1150.

2. T. Reck, C. Jung-Kubiak, J. V. Siles, C. Lee, R. Lin, G. Chattopadhyay, I. Mehdi, and K. Cooper, A silicon micromachined eight-pixel transceiver array for submillimeter-wave radar, IEEE Transactions on Terahertz Science and Technology 5 (2015), no. 2, 197-206.

3. U. Shah, E. Decrossas, C. Jung-Kubiak, T. Reck, G. Chattopadhyay, I. Mehdi, and J. Oberhammer, Submillimeter-wave 3.3-bit RF MEMS phase shifter integrated in micromachined waveguide, IEEE Transactions on Terahertz Science and Technology 6 (2016), no. 5, 706-715.

4. A. Gomez-Torrent, U. Shah, and J. Oberhammer, Compact silicon-micromachined wideband 220$330 \mathrm{GHz}$ turnstile orthomode transducer, IEEE Transactions on Terahertz Science and Technology 9 (2019), 38-46.

5. H. Yang, Y. Dhayalan, X. Shang, M. J. Lancaster, B. Liu, H. Wang, M. Henry, and P. G. Huggard, WR3 waveguide bandpass filters fabricated using high precision CNC machining and SU-8 photoresist technology, IEEE Transactions on Terahertz Science and Technology 8 (2018), no. 1, 100-107.

6. O. Glubokov, X. Zhao, J. Campion, U. Shah, and J. Oberhammer, Micromachined filters at $450 \mathrm{GHz}$ with $1 \%$ fractional bandwidth and unloaded $Q$ over 700 , IEEE Transactions on Terahertz Science and Technology 9 (2019), 106-108.

7. T. Reck, C. Jung-Kubiak, and G. Chattopadhyay, A 700-GHz MEMS waveguide switch, IEEE Transactions on Terahertz Science and Technology 6 (2016), no. 4, 641-643.

8. U. Shah, T. Reck, H. Frid, C. Jung-Kubiak, G. Chattopadhyay, I. Mehdi, and J. Oberhammer, A 500$750 \mathrm{GHz}$ RF MEMS waveguide switch, IEEE Transactions on Terahertz Science and Technology 7 (2017), no. 3, 326-334.

9. T. Kojima, A. Gonzalez, S. Asayama, and Y. Uzawa, Design and development of a hybrid-coupled waveguide multiplexer for a multiband receiver, IEEE Transactions on Terahertz Science and Technology 7 (2017), no. 1, 10-19.

10. C. A. Leal-Sevillano, K. B. Cooper, J. A. Ruiz-Cruz, J. R. Montejo-Garai, and J. M. Rebollar, A 225 $\mathrm{GHz}$ circular polarization waveguide duplexer based on a septum orthomode transducer polarizer, IEEE Transactions on Terahertz Science and Technology 3 (2013), no. 5, 574-583.

11. T. J. Reck, C. Jung-Kubiak, J. Gill, and G. Chattopadhyay, Measurement of silicon micromachined waveguide components at 500-750 GHz, IEEE Transactions on Terahertz Science and Technology 4 (2014), no. 1, 33-38. 
12. C. Jung-Kubiak, T. J. Reck, J. V. Siles, R. Lin, C. Lee, J. Gill, K. Cooper, I. Mehdi, and G. Chattopadhyay, A multistep DRIE process for complex terahertz waveguide components, IEEE Transactions on Terahertz Science and Technology 6 (2016), no. 5, 690-695.

13. J. Campion, O. Glubokov, A. Gomez, A. Krivovitca, U. Shah, L. Bolander, Y. Li, and J. Oberhammer, An ultra low-loss silicon-micromachined waveguide filter for D-band telecommunication applications. In: 2018 IEEE/MTT-S international microwave symposium-IMS. IEEE, pp. 583-586, 2018.

14. J. Svedin, R. Malmqvist, B. Beuerle, U. Shah, and J. Oberhammer, A 230-300 GHz low-loss micromachined waveguide hybrid coupler. In: 2017 47th European microwave conference (EuMC), pp. 616-619, 2017.

15. R. Malmqvist, A. Gustafsson, J. Svedin, B. Beuerle, U. Shah, and J. Oberhammer, A 220-325 GHz low-loss micromachined waveguide power divider. In: 2017 IEEE Asia Pacific Microwave Conference (APMC), pp. 291-294, 2017.

16. O. Glubokov, X. Zhao, J. Campion, B. Beuerle, U. Shah, and J. Oberhammer, Investigation of fabrication accuracy and repeatability of high- $Q$ silicon-micromachined narrowband sub-THz waveguide filters, IEEE Transactions on Microwave Theory and Techniques 67 (2019), no. 9, 3696-3706.

17. B. Beuerle, J. Campion, U. Shah, and J. Oberhammer, A very low loss 220-325 GHz silicon micromachined waveguide technology, IEEE Transactions on Terahertz Science and Technology 8 (2018), no. 2, 248-250.

18. J. Campion, A. Hassona, Z. S. He, B. Beuerle, A. Gomez-Torrent, U. Shah, S. Vecchiattini, R. Lindman, T. S. Dahl, Y. Li, H. Zirath, and J. Oberhammer, Toward industrial exploitation of $\mathrm{THz}$ frequencies: Integration of SiGe MMICs in silicon-micromachined waveguide systems, IEEE Transactions on Terahertz Science and Technology 9 (2019), no. 6, 624-636.

19. B. Beuerle, U. Shah, and J. Oberhammer, Micromachined waveguides with integrated silicon absorbers and attenuators at 220-325 GHz. In: 2018 IEEE/MTT-S international microwave symposium - IMS, pp. 291-294, 2018.

20. J. Campion, U. Shah, and J. Oberhammer, Elliptical alignment holes enabling accurate direct assembly of micro-chips to standard waveguide flanges at sub-THz frequencies. In: 2017 IEEE MTT-S international microwave symposium (IMS), pp. 1262-1265, 2017.

21. J. Campion, U. Shah, and J. Oberhammer, Silicon-micromachined waveguide calibration shims for terahertz frequencies. In: 2019 IEEE MTT-S international microwave symposium (IMS), pp. 12651268, 2019.

22. D. M. Pozar, Microwave engineering, 3rd ed., Wiley, Hoboken, 2004.

Publisher's Note Springer Nature remains neutral with regard to jurisdictional claims in published maps and institutional affiliations. 